\title{
CORRELAÇÕES E ANÁLISE DE TRILHA EM GIRASSOL (1)
}

\author{
EDSON PERITO AMORIM $\left({ }^{2}\right)$; NILZA PATRÍCIA RAMOS $\left({ }^{3}\right)$; \\ MARIA REGINA GONÇALVES UNGARO $\left({ }^{4}\right)$; TAMMY A. M. KIIHL $\left({ }^{4}\right)$
}

\section{RESUMO}

Catorze genótipos de girassol foram utilizados para estimar valores de correlação entre treze características agronômicas em Campinas (SP), Brasil. A análise de trilha foi empregada para particionar os valores de correlação em efeitos diretos e indiretos, sobre a produtividade de grãos. Valores de correlação positivos e significativos foram obtidos entre a produtividade de grãos e as características diâmetro do capítulo $(\mathrm{r}=0,63)$ e massa de mil grãos $(\mathrm{r}=0,55)$. O diâmetro do capítulo, a porcentagem de grãos normais e a massa de mil grãos tiveram efeitos diretos positivos sobre a produtividade de grãos, podendo ser utilizados na seleção indireta, devido à sua fácil medição.

Palavras-chave: germoplasma, seleção indireta, melhoramento de plantas, Helianthus annuus L.

\section{ABSTRACT \\ CORRELATIONS AND PATH ANALYSIS IN SUNFLOWER}

Possible correlations among grain yield and thirteen agronomic characteristics were investigated in fourteen sunflower germplasm in a field experiment in Campinas, Brazil. In order to better understand the interrelationships among the studied variables path coefficient analysis was used for partitioning correlation in direct and indirect effects. Significant positive correlations were observed between grain yield and head diameter $(r=0.63)$, and weight of 1000 grains $(r=0.55)$. Head diameter, percentage of normal grains and weight of 1000 grains can be easily measured and showed positive direct effects on grain yield which led them suitable for indirect selection.

Key words: germplasm, indirect selection, plant breeding, Helianthus annuus L.

$\left({ }^{1}\right)$ Recebido para publicação em 7 de dezembro de 2006 e aceito em 6 de novembro de 2007.

$\left(^{2}\right)$ Embrapa Mandioca e Fruticultura Tropical. Rua Embrapa, s/nº. Caixa Postal 007, 44380-000 Cruz das Almas (BA). E-mail: edson@cnpmf.embrapa.br $\left(^{*}\right)$ Autor correspondente.

$\left({ }^{3}\right)$ Embrapa Meio Ambiente. Rodovia SP 340 - km 127,5 Caixa Postal 69, 13820-000 Jaguariúna (SP). E-mail: npramos@cnpma.embrapa.br.

$\left.{ }^{4}\right)$ Instituto Agronômico (IAC), Centro de Análise e Pesquisa Tecnológica do Agronegócio de Grãos e Fibras, Caixa Postal 28, 13012-970 Campinas (SP). E-mail: ungaro@iac.sp.gov.br, tammy@yahoo.com.br. 


\section{INTRODUÇÃO}

O girassol é cultivado no Brasil, predominantemente, após a colheita da safra de verão, em sucessão à soja ou milho. De acordo com LAzZAROTto et al. (2005), este cultivo em "safrinha" permite aos produtores utilizar a maior parte dos recursos disponíveis na propriedade, entre eles: mãode-obra e máquinas, que poderiam ficar ociosos em um determinado período do ano. Para a expansão do cultivo do girassol, há necessidade de adequá-lo aos diferentes sistemas de produção das culturas tradicionais, como milho, soja, cana-de-açúcar, arroz e outras (Oliveira et al., 2005). Ainda, segundo os autores, os programas de melhoramento genético devem procurar desenvolver genótipos de girassol que tenham concomitantemente: alto teor de óleo, ciclo precoce, porte baixo, resistência a fatores bióticos e abióticos, além de alta produtividade.

A produtividade de grãos é um caráter complexo, resultante da expressão e da associação de diferentes componentes, que são considerados pelo melhorista no processo de seleção de novos genótipos. Para que a seleção seja realizada de forma eficiente, são necessárias informações sobre a natureza e a magnitude das variações fenotípicas observadas em uma determinada população, bem como sobre as correlações de outras características agronômicas com a produtividade, ou mesmo entre elas, e sobre a extensão da influência ambiental na expressão das características estudadas (Gomes et al., 2007).

A correlação fenotípica entre duas características é governada pelos componentes genético e ambiental. A correlação genética é causada pela presença de pleiotropia e/ou desequilíbrio de ligação. A correlação fenotípica é a única onde a observação direta do fenômeno é possível (FALCONER e MACKAY, 1996).

A correlação quantifica a associação entre duas variáveis quaisquer. Portanto, não permite inferências sobre causa e efeito, impossibilitando o conhecimento de qual tipo de associação governa o par de caracteres (FURTADO et al., 2002). A análise de trilha, proposta por WRIGHT (1921), permite particionar o coeficiente de correlação em efeitos diretos e indiretos (coeficiente de trilha). Para CRUZ e CARNeIRO (2003) esta análise pode ser definida como um coeficiente de regressão padronizado, sendo uma expansão da análise de regressão múltipla, quando estão envolvidos inter-relacionamentos complexos.

Em girassol, alguns trabalhos têm sido realizados com o objetivo de estimar a correlações entre diferentes características agronômicas, assim como decompô-las em seus efeitos diretos e indiretos, por meio da análise de trilha (JокSIMOVIC et al., 1999; TEKLEWOLD et al., 2000; CHIKKADEVAIAH et al., 2002; WANI, 2004; Manivannan et al. 2005; Vidhyavathi et al., 2005; Farhatullah e Khalil, 2006 e Hladni et al., 2006).

Os objetivos da presente pesquisa foram estimar, para as condições brasileiras, as correlações genéticas entre treze caracteres agronômicos e seus efeitos diretos e indiretos, por meio da análise de trilha, sobre a produtividade de grãos em 14 genótipos de girassol, visando subsidiar programas de melhoramento nos processos de seleção de genótipos promissores.

\section{MATERIAL E MÉTODOS}

Foram avaliados 14 genótipos de girassol pertencentes a diferentes programas de melhoramento genético. Cada genótipo foi semeado em uma parcela de 4 linhas de 6,0 $\mathrm{m}$ de comprimento, espaçadas de $0,8 \mathrm{~m}$ entre linhas e $0,3 \mathrm{~m}$ entre plantas. Somente as duas linhas centrais foram consideradas como parcelas úteis para as avaliações. Foi realizado desbaste aos sete dias após a emergência, deixandose uma planta por cova, e a adubação realizada foi a recomendada para a cultura (QUAGGIO e Ungaro, 1997). O experimento foi instalado no Centro Experimental Central, do Instituto Agronômico, localizado no município de Campinas, Estado de São Paulo, na safra 2006.

O delineamento estatístico utilizado foi o de blocos casualizados com quatro repetições e as seguintes características agronômicas foram mensuradas:

1. Início do florescimento (IF, em dias): anotado no momento do aparecimento da primeira flor no estádio fenológico R4 (CONNOR e HALL, 1997);

2. $50 \%$ do florescimento ( $\mathrm{F}$, em dias): anotado quando $50 \%$ das plantas da parcela útil estavam no estádio fenológico R4;

3. Número de folhas (NF): observado em cinco plantas competitivas, quando $50 \%$ das plantas da parcela útil estavam no estádio fenológico R4;

4. Altura da planta (AP, em $\mathrm{cm}$ ): medida da base do solo até a inserção do capítulo, em cinco plantas competitivas da parcela útil;

5. Altura da inserção do capítulo $(\mathrm{AC}, \mathrm{em} \mathrm{cm})$ : medida da base do solo até o ponto central do capítulo, em cinco plantas competitivas da parcela útil;

6. Diâmetro da haste ( $\mathrm{DH}$, em $\mathrm{mm})$ : medido a $10 \mathrm{~cm}$ do solo em cinco plantas competitivas da parcela útil; 
7. Diâmetro do capítulo (DC, em $\mathrm{cm})$ : média de cinco capítulos de cada parcela útil;

8. Curvatura do capítulo (CC): avaliação visual e classificação segundo KNowLEs (1978) de cinco plantas na parcela;

9. Porcentagem de grãos normais (GN): obtida pela diferença entre o número de grãos normais e o número de chochos, dividida pelo número total de grãos; média de cinco capítulos de cada parcela útil;

10. Massa de mil grãos (MG, em g): obtido pela razão entre o massa do total de grãos de cada um dos cinco capítulos de cada parcela útil pelo número total de grãos, sendo posteriormente corrigido para mil grãos;

11. Peso hectolítrico ( $\mathrm{PH}$, em kg $\left.100 \mathrm{l}^{-1}\right)$ : seguindo método proposto por BRASIL (1992);

12. Produtividade de grãos (PROD, em kg ha' $\left.{ }^{1}\right)$ : média de 36 plantas da parcela útil.

13. Teor de óleo (TO, em \%): seguindo o método de ressonância magnética nuclear, proposto por International Union Of Pure And Applied Chemistry (1987).

Para cada caráter, foi feita a análise de variância e as médias foram comparadas pelo teste de ScotT e KnotT (1974) a 5\%. Para fins de comparações múltiplas, os efeitos de genótipos foram considerados como fixos. As estimativas das correlações genéticas foram obtidas como descrito por STEEL e TORRIE (1980). Realizou-se o desdobramento dessas correlações em efeitos diretos e indiretos das 12 características agronômicas sobre a produtividade de grãos (PROD), por meio da análise de trilha, descrita por CRUZ e CARneiro (2003).

O nível de multicolinearidade da matriz singular $X^{\prime} X$ foi estabelecido pelo produto do respectivo elemento da diagonal de $X^{\prime} X$ pelo componente de variância residual. A variância será tanto maior quanto maior for a magnitude deste elemento diagonal, denominado fator de inflação da variância (FIV), segundo MARQUARDT (1970). Para atenuar o efeito da variância muito alta, o sistema de equações normais foi modificado, pela adição de uma constante $k$ aos valores dos elementos da diagonal da matriz de acordo com Hoerl e Kennard (1970a e 1970b). CARVAlHo et al. (2002) ressalta que deve ser escolhido um valor de $k$ para o qual a maioria dos coeficientes de trilha esteja estabilizada.

O valor do coeficiente foi estabelecido por meio de gráficos, nos quais foram plotados os valores dos efeitos diretos das características em função dos valores de $k$ Cruz e Carneiro (2003). De acordo com Neter et al. (1974), a existência de pelo menos um FIV com valor superior a 10, é um indicativo de que os coeficientes de regressão associados a estes valores tenham estimativas influenciadas pela multicolinearidade.

Todas as análises foram realizadas utilizandose o aplicativo estatístico-computacional Genes (CRUZ, 1997).

\section{RESULTADOS E DISCUSSÃO}

Foram observados efeitos significativos da fonte de variação genótipos para todas as características, indicando a existência de variabilidade genética entre eles para os caracteres avaliados (Tabela 1). Os coeficientes de variação experimental apresentaram magnitudes variando de 2,84\% para F a 19,38\% para PROD (Tabela 1 ).

Com base nos resultados do teste de médias realizado para o caráter IF, foi possível identificar genótipos precoces (BRSG03, BRSG08, Catissol e Embrapa122) e tardios (M734, V20038, V20044, VDH487 e ACA861). Além disso, nota-se que os genótipos pertencentes a programas brasileiros de melhoramento (série BRSG, Catissol e Embrapa122) são aqueles mais precoces comparativamente aos genótipos de programas de melhoramento argentinos (ACA861, EXP1447, M734, V20038, V20044 e VDH487). Exceção feita ao híbrido triplo AGROBEL960, de origem argentina, de comportamento intermediário quanto aos caracteres F e IF. Cabe destacar que os programas brasileiros buscam selecionar genótipos precoces (Oliveira et al., 2005), visando aproveitar a entressafra das grandes culturas, fato ainda pouco explorado na Argentina, onde o girassol é cultivado como cultura principal e seu ciclo varia de médio a tardio (Romano e VÁzquez, 2003).

Para os caracteres AP, AC, DH, DC, CC, GN, MG e PH observou-se a formação de diferentes agrupamentos, de acordo com o teste de SCOTT e KNOTT (1974). As médias para IF, NF, AP, DH, DC e MG estão dentro do intervalo constatado por CASTRO e FARIAS (2005). A média geral de CC foi de 3,7 , semelhante à média 3,9 observada em ensaios de girassol desenvolvidos na mesma região (EMBRAPA, 1997). A altura da inserção do capítulo foi, em média, de 115 $\mathrm{cm}$ abaixo da observada para Campinas na safra 2002/2003, de 158 cm (EMBRAPA, 2003), confirmando o esperado, uma vez que os dados deste ensaio foram obtidos na "safrinha", época de menor insolação e disponibilidade hídrica. 


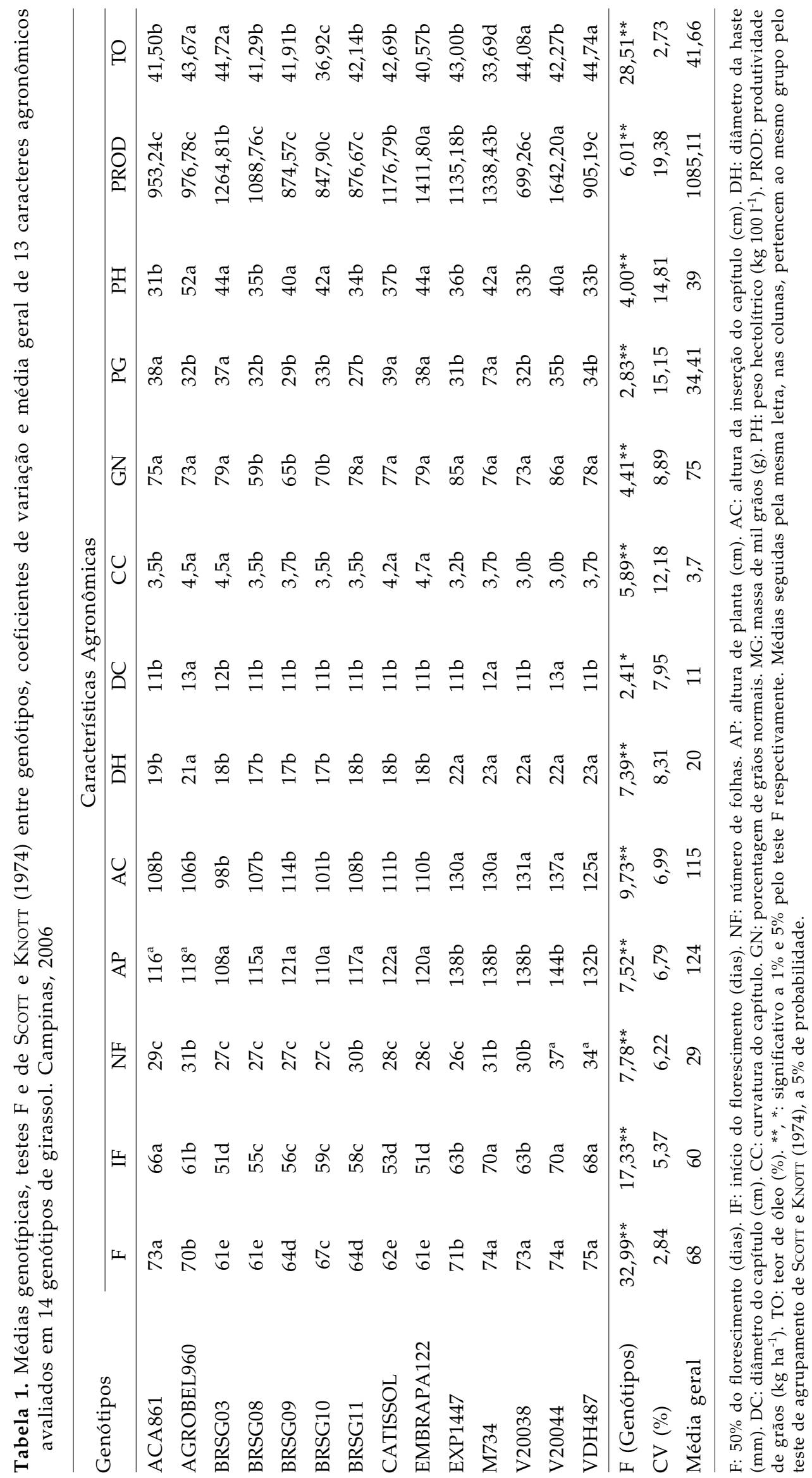


O teor de óleo variou de 33,69\% para o híbrido M734 a 44,74\% para o híbrido VDH487. A média geral do experimento ficou em $41,66 \%$. Estes valores estão dentro dos limites de variação dessa característica (28\%-60\%), de acordo com CASTRO e FARIAS (2005), em revisão realizada sobre a variação de características agronômicas em girassol.

A produtividade de grãos teve média de $1085,11 \mathrm{~kg} \mathrm{ha}^{-1}$, variando de $699,26 \mathrm{~kg} \mathrm{ha}^{-1}$ para o híbrido V20038 a $1642,20 \mathrm{~kg} \mathrm{ha}^{-1}$ para o híbrido V20044. Observou-se nas variedades produtividade média de $1284,46 \mathrm{~kg} \mathrm{ha}^{-1}, 25 \%$ superior à média dos híbridos, que ficou em $1030,74 \mathrm{~kg} \mathrm{ha}^{-1}$. Esses valores estão em concordância com aqueles verificados na literatura (EMBRAPA, 2003; 2006a).

O peso hectolítrico teve média de $39 \mathrm{~kg} 100 \mathrm{l}^{-1}$, a qual está contida no intervalo observado por CARTER

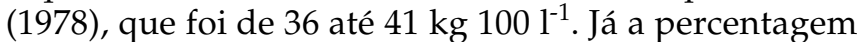
de grãos normais (GN) teve média de $75 \%$, podendo ser considerada alta em função do déficit hídrico ocorrido durante o período do ensaio. A precipitação pluvial acumulada entre março e julho de 2006 foi de $290 \mathrm{~mm}$, enquanto a série histórica (1961-1990) indica valores em torno de $370 \mathrm{~mm}$ para o mesmo período (EMBRAPA, 2006b). Durante o período de enchimento de grãos (maio-junho), a precipitação acumulada não ultrapassou $60 \mathrm{~mm}$, enquanto na fase de formação do botão floral, em abril, o total de chuvas ficou em $27 \mathrm{~mm}$, períodos estes considerados críticos para a produtividade em grãos (Gomes et al., 2004). De acordo com CASTRO e FARIAS (2005), 400 a $500 \mathrm{~mm}$ de água, bem distribuídos ao longo do ciclo, resultam em rendimentos próximos ao potencial máximo. A condição climática observada no presente experimento facilitou a identificação, por meio da produtividade de grãos, dos genótipos com maiores níveis de tolerância à seca. Entre os mais tolerantes, destacam-se a variedade Embrapa 122 e o híbrido V20044; os genótipos sensíveis foram V20038, VDH487, BRSG08, BRSG9, BRSG10, BRSG11, AGROBEL960 e ACA861 (Tabela 1).

Cabe destacar que é possível identificar genótipos com características agronômicas complementares, como por exemplo: o híbrido simples V20044 (tardio, alta produtividade de grãos, elevada AP e tolerante ao estresse hídrico) e Embrapa 122 (precoce em relação ao IF, porte baixo, alta produtividade de grãos e também tolerante à seca) (Tabela 1). Esses genótipos poderiam ser utilizados em esquemas de hibridação e/ou seleção, com o objetivo de incorporar as características favoráveis de cada parental em uma mesma população, ou mesmo de obter novas linhagens a partir destes genótipos. De acordo com AMORIM e SouZA (2005), a extração de linhagens a partir de variedades melhoradas e/ou híbridos comerciais é uma alternativa viável, pois estes genótipos foram testados em vários ambientes e, no caso dos híbridos, ainda é possível contar com grande proporção de alelos favoráveis já fixados.

Estimativas altas, positivas e significativas do coeficiente de correlação foram observadas entre $\mathrm{F}$ e os caracteres IF $(r=0,97)$, NF $(r=0,72)$, AP $(r=0,72)$, AC $(r=0,73)$ e DH $(r=0,84)$. As associações entre NF e os caracteres AP, AC e DH foram positivas e significativas $(\mathrm{r}=0,58, \mathrm{r}=0,59$ e $\mathrm{r}=0,73$ respectivamente), de forma semelhante ao observado entre DH e AP $(r=0,84)$ e DH e AC $(r=0,82)$. Em DH também houve correlação alta e significativa com o NF $(r=0,73)$. Esses resultados concordam com os observados por TeKLEWOLD et al. (2000), KHAN (2001) e HASSAN (2001).

No peso hectolítrico houve correlação significativa com o DC $(r=0,59)$ sugerindo que quanto maior o tamanho do capítulo maior será o PH (Tabela 2). De acordo com CASTRO e FARIAS (2005), capítulos bem desenvolvidos tendem a ter maior proporção de aquênios grandes e mais pesados. Para Alkio et al. (2003) esses aquênios têm mais tempo para o enchimento, possibilitando maior aporte de nutrientes.

A ausência de correlação entre IF e PROD e entre F e PROD (Tabela 2) sugere que a duração da atividade fotossintética não é uma limitação em girassol, no que se refere à produtividade. Desta forma, a redução no ciclo da planta, pelo melhoramento genético, não está associada à redução na produtividade, o que é muito importante quando se objetiva obter cultivares precoces e altamente produtivos. Outros autores constataram resultados semelhantes (PATIL et al., 1996; DodDamani et al., 1997).

A produtividade de grãos (PROD) revelou correlação positiva significativa com o DC $(r=0,63)$ e com o MG $(r=0,55)$ (Tabela 2). Esses resultados corroboram com os verificados por LAKSHAMANRAO et al. (1985), Tyagi (1985), Hladni et al. (2006) e Farhatullah e Khalil (2006). Diante deste fato, a seleção de material com maior diâmetro do capítulo e/ou massa de mil grãos, permitirá a obtenção de genótipos com boa produtividade. Tanto o DC quanto o PG são características de fácil mensuração, sendo úteis na seleção indireta para produtividade.

No caráter teor de óleo não se observou correlação significativa com nenhuma das características, destacando-se que a correlação de TO com PROD, MG e PH foi baixa e negativa $(\mathrm{r}=-0,20, \mathrm{r}=-$ 0,42 e $\mathrm{r}=-0,18$, respectivamente). HLADNI et al. (2006) observaram valores de correlação negativa entre teor de óleo e massa de mil grãos, e produtividade $(\mathrm{r}=-0,85$ e $\mathrm{r}=-0,71$ respectivamente). TYAGI (1985) também constatou correlação negativa entre teor de óleo e produtividade $(r=-0,19)$. 


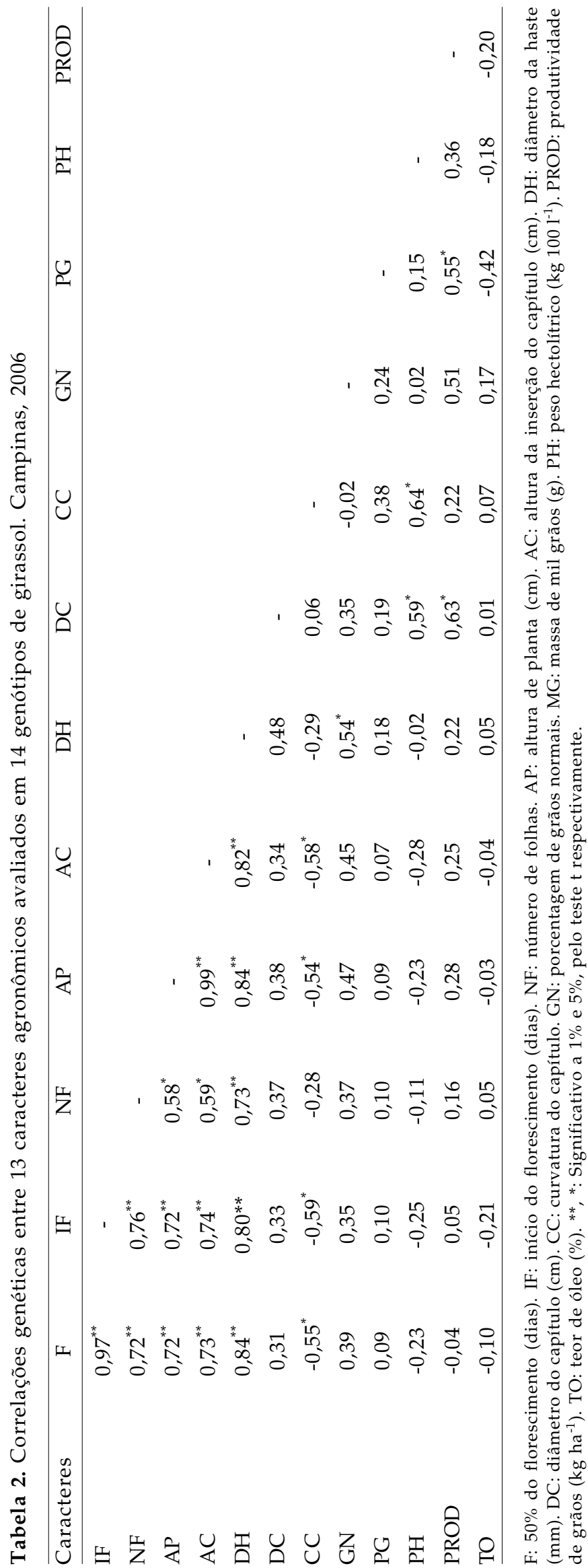

De acordo com CASTRO e FARIAS (2005) existe uma dinâmica contrária entre o teor de óleo e o tamanho e massa de mil aquênios. Para os autores, os aquênios formados na periferia do capítulo são maiores e mais pesados que os formados no centro do capítulo, embora tenham menor teor. Esse ocorre porque os aquênios maiores, localizados na periferia, têm maior volume e superfície de casca e, por conseqüência, menor teor de óleo que aquênios formados no centro do capítulo.

O diagnóstico de multicolinearidade obtido a partir da escolha do coeficiente $k$ igual a 0,05 permitiu a correção das distorções, podendo-se observar valores de FIV sempre menores que 10, com maior confiabilidade nas interpretações de causa e efeito entre os caracteres estudados (Tabela 3).

O coeficiente de determinação revela que $82 \%$ da PROD pode ser explicada pelo efeito das variáveis analisadas (Tabela 3). Cabe salientar que esse valor se restringe aos caracteres em análise, em função da PROD ser um caráter quantitativo, com grande número de alelos de pequeno efeito (modificadores) influenciando o caráter; com variância ambiental marcante e por conseqüência, herdabilidade baixa.

Todas as variáveis influenciaram a PROD, e em algumas delas observaram-se correlaçóes significativas entre si (Tabela 2). Permite-se inferir que ocorre um sistema de inter-relações entre estes caracteres e determinada variável poderia exercer influência na PROD por meio de outra característica correlacionada, caracterizando os efeitos indiretos dos caracteres analisados sobre PROD (Tabela 3).

As magnitudes dos efeitos diretos dos caracteres analisados sobre PROD foram inferiores às magnitudes das estimativas das suas respectivas correlações com PROD (Tabela 3). Com base nessa informação, é possível inferir que existem outras características influenciando tanto a magnitude quanto o sentido da correlação entre F e PROD.

O diâmetro do capítulo (DC) apresentou efeito direto sobre a PROD $(0,58)$. Esse resultado concorda com aqueles constatados por AsHOK et al. (2000) e FARHATULLAH e KHALIL (2006). O presente resultado permite inferir que o efeito direto do DC sobre a PROD é positivo, sendo a correlação genética entre estas características alta e significativa $(r=0,63)$ fazendo com que genótipos com maior produtividade de grãos possam ser obtidos a partir da seleção para maior diâmetro de capítulo.

A porcentagem de grãos normais $(\mathrm{GN})$ tem efeito direto positivo sobre a PROD $(0,36)$, e efeito indireto via diâmetro do capítulo $(0,20)$ e $\mathrm{F}(-0,24)$. Comportamento semelhante foi observado para o caráter massa de mil grãos (MG), com efeito direto positivo sobre PROD $(0,34)$, embora o efeito indireto de PG sobre PROD tenha sido menor $(0,10)$. 
Tabela 3. Estimativas de efeitos diretos e indiretos de 12 características agronômicas sobre a produtividade de grãos (PROD) em 14 genótipos de girassol, obtidos pelo método da análise de trilha, com diagnóstico de multicolinearidade. Campinas, 2006

\begin{tabular}{|c|c|c|c|c|c|}
\hline Característica & $\mathrm{R}$ & FIV & & $\mathrm{r}$ & FIV \\
\hline \multicolumn{6}{|c|}{$50 \%$ do florescimento $(\mathrm{F})$} \\
\hline ED sobre PROD & $-0,63$ & 9,65 & EI via DC & 0,18 & 0,34 \\
\hline EI via IF & 0,04 & 9,82 & EI via CC & 0,01 & 1,55 \\
\hline EI via NF & 0,08 & 1,27 & EI via GN & 0,14 & 0,23 \\
\hline EI via AP & 0,02 & 5,56 & EI via PG & 0,03 & 0,02 \\
\hline EI via AC & 0,17 & 5,83 & EI via PH & 0,02 & 0,28 \\
\hline EI via $\mathrm{DH}$ & $-0,10$ & 4,77 & EI via TO & 0,02 & 0,02 \\
\hline $\mathrm{r}_{\mathrm{g}}$ & $-0,04$ & & & & \\
\hline \multicolumn{6}{|c|}{ Início do florescimento (IF) } \\
\hline ED sobre PROD & 0,04 & 9,86 & EI via DC & 0,19 & 0,39 \\
\hline EI via $F$ & $-0,61$ & 8,29 & EI via CC & 0,02 & 1,79 \\
\hline EI via NF & 0,08 & 1,42 & EI via GN & 0,12 & 0,18 \\
\hline EI via AP & 0,02 & 5,52 & EI via PG & 0,03 & 0,03 \\
\hline EI via AC & 0,17 & 5,92 & EI via $\mathrm{PH}$ & 0,02 & 0,35 \\
\hline EI via $\mathrm{DH}$ & $-0,09$ & 4,34 & EI via TO & 0,04 & 0,11 \\
\hline $\mathrm{r}_{\mathrm{g}}$ & 0,05 & & & & \\
\hline \multicolumn{6}{|c|}{ Número de folhas (NF) } \\
\hline ED sobre PROD & 0,11 & 2,72 & EI via DC & 0,21 & 0,49 \\
\hline EI via F & $-0,04$ & 4,52 & EI via CC & 0,01 & 0,40 \\
\hline EI via IF & 0,03 & 6,38 & EI via GN & 0,14 & 0,20 \\
\hline EI via AP & 0,02 & 3,58 & EI via PG & 0,03 & 0,02 \\
\hline EI via AC & 0,13 & 3,70 & EI via $\mathrm{PH}$ & 0,01 & 0,07 \\
\hline EI via $\mathrm{DH}$ & $-0,08$ & 3,61 & EI via TO & $-0,01$ & 0,01 \\
\hline $\mathrm{r}_{\mathrm{g}}$ & 0,16 & & & & \\
\hline \multicolumn{6}{|c|}{ Altura de planta (AP) } \\
\hline ED sobre PROD & 0,03 & 9,95 & EI via DC & 0,22 & 0,51 \\
\hline EI via $\mathrm{F}$ & $-0,46$ & 4,57 & EI via CC & 0,01 & 1,48 \\
\hline EI via IF & 0,03 & 5,75 & EI via GN & 0,17 & 0,33 \\
\hline EI via NF & 0,06 & 0,81 & EI via PG & 0,03 & 0,02 \\
\hline EI via AC & 0,23 & 9,82 & EI via $\mathrm{PH}$ & 0,02 & 0,29 \\
\hline EI via $\mathrm{DH}$ & $-0,10$ & 4,76 & EI via TO & 0,00 & 0,00 \\
\hline $\mathrm{r}_{\mathrm{g}}$ & 0,28 & & & & \\
\hline \multicolumn{6}{|c|}{ Altura da inserção do capítulo (AC) } \\
\hline ED sobre PROD & 0,23 & 9,88 & EI via DC & 0,20 & 0,41 \\
\hline EI via F & $-0,46$ & 4,72 & EI via CC & 0,02 & 1,45 \\
\hline EI via IF & 0,03 & 6,05 & EI via GN & 0,16 & 0,29 \\
\hline EI via NF & 0,06 & 0,85 & EI via PG & 0,02 & 0,01 \\
\hline EI via AP & 0,03 & 9,93 & EI via PH & 0,03 & 0,44 \\
\hline EI via $\mathrm{DH}$ & $-0,09$ & 4,56 & EI via TO & 0,01 & 0,00 \\
\hline $\mathrm{r}_{\mathrm{g}}$ & 0,25 & & & & \\
\hline \multicolumn{6}{|c|}{ Diâmetro da haste (DH) } \\
\hline ED sobre PROD & $-0,12$ & 7,46 & EI via DC & 0,27 & 0,80 \\
\hline EI via $\mathrm{F}$ & $-0,53$ & 6,18 & EI via CC & 0,01 & 0,42 \\
\hline EI via IF & 0,03 & 7,12 & EI via GN & 0,19 & 0,43 \\
\hline EI via NF & 0,08 & 1,32 & EI via PG & 0,06 & 0,07 \\
\hline EI via AP & 0,02 & 7,50 & EI via $\mathrm{PH}$ & 0,00 & 0,00 \\
\hline EI via $A C$ & 0,19 & 7,30 & EI via TO & $-0,01$ & 0,01 \\
\hline$r_{g}$ & 0,22 & & & & \\
\hline
\end{tabular}


Quadro 3. Conclusão

\begin{tabular}{|c|c|c|c|c|c|}
\hline Característica & $\mathrm{R}$ & FIV & & $\mathrm{r}$ & FIV \\
\hline \multicolumn{6}{|l|}{ Diâmetro do capítulo (DC) } \\
\hline ED sobre PROD & 0,58 & 3,84 & EI via $\mathrm{DH}$ & $-0,06$ & 1,53 \\
\hline EI via $\mathrm{F}$ & $-0,19$ & 0,85 & EI via CC & $-0,00$ & 0,02 \\
\hline EI via IF & 0,01 & 1,23 & EI via GN & 0,13 & 0,18 \\
\hline EI via NF & 0,04 & 0,34 & EI via PG & 0,06 & 0,08 \\
\hline EI via $\mathrm{AP}$ & 0,01 & 1,55 & EI via $\mathrm{PH}$ & $-0,06$ & 1,95 \\
\hline EI via $\mathrm{AC}$ & 0,08 & 1,28 & EI via TO & $-0,00$ & 0,00 \\
\hline $\mathrm{r}_{\mathrm{g}}$ & 0,63 & & & & \\
\hline \multicolumn{6}{|l|}{ Curvatura do capítulo (CC) } \\
\hline ED sobre PROD & $-0,03$ & 5,69 & EI via $\mathrm{DH}$ & 0,03 & 0,55 \\
\hline EI via $\mathrm{F}$ & 0,34 & 2,64 & EI via DC & 0,03 & 0,01 \\
\hline EI via IF & $-0,02$ & 3,86 & EI via GN & $-0,00$ & 0,00 \\
\hline EI via NF & $-0,03$ & 0,19 & EI via PG & 0,13 & 0,35 \\
\hline EI via AP & $-0,02$ & 3,06 & EI via $\mathrm{PH}$ & $-0,06$ & 2,29 \\
\hline EI via $A C$ & $-0,13$ & 3,68 & EI via TO & $-0,01$ & 0,01 \\
\hline $\mathrm{r}_{\mathrm{g}}$ & 0,22 & & & & \\
\hline \multicolumn{6}{|c|}{ Porcentagem de grãos normais (GN) } \\
\hline ED sobre PROD & 0,36 & 1,63 & EI via $\mathrm{DH}$ & $-0,06$ & 1,98 \\
\hline EI via $\mathrm{F}$ & $-0,24$ & 1,34 & EI via DC & 0,20 & 0,42 \\
\hline EI via IF & 0,01 & 1,36 & EI via CC & 0,00 & 0,00 \\
\hline EI via NF & 0,04 & 0,34 & EI via PG & 0,08 & 0,14 \\
\hline EI via AP & 0,02 & 2,38 & EI via $\mathrm{PH}$ & $-0,00$ & 0,00 \\
\hline EI via $\mathrm{AC}$ & $-0,10$ & 2,15 & EI via TO & $-0,03$ & 0,07 \\
\hline $\mathrm{r}_{\mathrm{g}}$ & 0,51 & & & & \\
\hline \multicolumn{6}{|l|}{ Massa de 1000 grãos (PG) } \\
\hline ED sobre PROD & 0,34 & 2,66 & EI via $\mathrm{DH}$ & $-0,02$ & 0,21 \\
\hline EI via $F$ & $-0,05$ & 0,07 & & EI via DC & 0,10 \\
\hline \multicolumn{6}{|l|}{0,12} \\
\hline EI via IF & 0,00 & 0,11 & EI via CC & $-0,01$ & 0,74 \\
\hline EI via NF & 0,01 & 0,03 & EI via GN & 0,08 & 0,08 \\
\hline EI via AP & 0,00 & 0,09 & EI via $\mathrm{PH}$ & $-0,02$ & 0,12 \\
\hline EI via $\mathrm{AC}$ & 0,02 & 0,05 & EI via TO & 0,07 & 0,45 \\
\hline $\mathrm{r}_{\mathrm{g}}$ & 0,55 & & & & \\
\hline \multicolumn{6}{|l|}{ Massa hectolítrico (PH) } \\
\hline ED sobre PROD & $-0,10$ & 6,09 & EI via $\mathrm{DH}$ & 0,00 & 0,00 \\
\hline EI via $F$ & 0,14 & 0,45 & EI via DC & 0,34 & 1,24 \\
\hline EI via IF & $-0,01$ & 0,71 & EI via CC & $-0,01$ & 2,14 \\
\hline EI via NF & $-0,01$ & 0,03 & EI via GN & 0,00 & 0,00 \\
\hline EI via AP & $-0,01$ & 0,56 & EI via PG & 0,05 & 0,05 \\
\hline EI via $\mathrm{AC}$ & $-0,06$ & 0,86 & EI via TO & 0,30 & 0,07 \\
\hline $\mathrm{r}_{\mathrm{g}}$ & 0,36 & & & & \\
\hline \multicolumn{6}{|l|}{ Teor de Óleo (TO) } \\
\hline ED sobre PROD & $-0,17$ & 2,84 & EI via $\mathrm{DH}$ & $-0,00$ & 0,02 \\
\hline EI via $F$ & 0,06 & 0,08 & EI via DC & 0,00 & 0,00 \\
\hline EI via IF & $-0,01$ & 0,49 & EI via CC & $-0,00$ & 0,02 \\
\hline EI via NF & 0,00 & 0,00 & EI via GN & 0,06 & 0,04 \\
\hline EI via AP & $-0,00$ & 0,01 & EI via PG & $-0,14$ & 0,43 \\
\hline EI via $\mathrm{AC}$ & $-0,00$ & 0,01 & EI via $\mathrm{PH}$ & 0,02 & 0,17 \\
\hline $\mathrm{r}_{\mathrm{g}}$ & $-0,20$ & & & & \\
\hline Coeficiente de determinação & 0,82 & & & & \\
\hline Valor de $\mathrm{k}$ & 0,05 & & & & \\
\hline Efeito da variável residual & 0,42 & & & & \\
\hline
\end{tabular}

ED: efeito direto. EI: efeito indireto. FIV: fator de inflação da variância. $\mathrm{r}_{\mathrm{g}}$ : correlação genética. F: $50 \%$ do florescimento (dias). IF: início do florescimento (dias). NF: número de folhas. AP: altura de planta $(\mathrm{cm})$. AC: altura da inserção do capítulo (cm). DH: diâmetro da haste (mm). DC: diâmetro do capítulo $(\mathrm{cm})$. CC: curvatura do capítulo. GN: porcentagem de grãos normais. MG: massa de mil grãos (g). PH: peso hectolítrico ( $\left.\mathrm{kg} 100 \mathrm{l}^{-1}\right)$. PROD: produtividade de grãos $\left(\mathrm{kg} \mathrm{ha}^{-1}\right)$. TO: teor de óleo (\%). 
O peso hectolítrico $(\mathrm{PH})$ promoveu efeito direto negativo sobre PROD $(-0,10)$, embora seu efeito indireto sobre PROD, via DC e TO, tenham sido positivos (0,34 e 0,30, respectivamente). Em relação ao teor de óleo, verificou-se efeito direto negativo sobre a PROD $(-0,17)$, concordando com a correlação observada entre estes dois caracteres $(r=-0,20)$.

Os resultados deste trabalho permitem inferir sobre a possibilidade da seleção indireta para produtividade de grãos utilizando como referência as características agronômicas diâmetro do capítulo, porcentagem de grãos normais e massa de mil grãos.

\section{CONCLUSÕES}

1. Existe variabilidade genética para todas as características analisadas, permitindo a seleção de genótipos superiores em populações segregantes ou mesmo em linhagens, obtidas a partir da hibridação destes genótipos de girassol.

2. Correlações significativas foram observadas entre a produtividade de grãos e os caracteres diâmetro do capítulo e massa de mil grãos.

3. O diâmetro do capítulo, a porcentagem de grãos normais e a massa de mil grãos proporcionaram efeito direto positivo sobre a produtividade de grãos, podendo também ser utilizados na seleção indireta visando ao desenvolvimento de novos genótipos com alto potencial produtivo.

\section{AGRADECIMENTOS}

Ao técnico de apoio à pesquisa Amarildo Cândido da Silva e as auxiliares de campo Sandra Cristina Leite de Oliveira e Lindaura Rodrigues de Carvalho, pelo manejo do experimento e colaboração na obtenção dos dados.

\section{REFERÊNCIAS}

ALKIO, M.; SCHUBERT, A.; DIEPENBROCK, W.; GRIMM, E. Effect of source-sink ratio on seed set and filling in sunflower (Helianthus annuus L.). Plant, Cell and Environment, Oxford, v.26, n.10, p.1609-1619, 2003.

AMORIM, E. P.; SOUZA, J. C. Híbridos de milho inter e intrapopulacionais obtidos a partir de populações $S_{0}$ de híbridos simples comerciais. Bragantia, Campinas, v.64, n.4, p.561-567, 2005.

ASHOK, S.; SHERRIFF, N. M.; NARAYANAN, S. L. Character association and path analysis in sunflower (Helianthus annuus L.). Crop Research, Hisar, v.20, p.453-456, 2000.
BRASIL, Ministério da Agricultura e Reforma Agrária. Regras para análise de sementes. Brasília: AGIPLAN, 1992. 365p.

CARTER, J. F. Sunflower science and technology. Madison: American Society of Agronomy. 1978. 505p. (Series Agronomy, 19).

CARVALHO, C. G.P.; ARIAS, C. A. A.; TOLEDO, J. F. F.; OLIVEIRA, M. F.; VELLO, N. A. Correlações e análise de trilha em linhagens de soja semeadas em diferentes épocas. Pesquisa Agropecuária Brasileira, Brasília, n.3, v.37, p.311-320, 2002.

CASTRO, C.; FARIAS, J. R. B. Ecofisiologia do girassol. In: LEITE, R. M. V. B.; BRIGHENTI, A. M.; CASTRO, C. (Ed). Girassol no Brasil. Londrina: Embrapa Soja, 2005. p.163-218.

CHIKKADEVAIA, H.; SUJATHA, H. L.; NANDINI, C. Correlation and path analysis in sunflower. Helia, Novi Sad, v.25,n.36, p.109-117, 2002.

CONNOR, J. D.; HALL, A. J. Sunflower physiology. In: SCHNEIDER, A. A. (Ed). Sunflower technology and production. Madison: ASA:CSSA:SSSA, 1997. p.113-181. (Series of Monographs, 35).

CRUZ, C. D. GENES: aplicativo computacional em genética e estatística. Viçosa: UFV, 1997. 442p.

CRUZ, C. D.; CARNEIRO, P. C. S. Modelos biométricos aplicados ao melhoramento genético. Viçosa: UFV, 2003. 585p.

DODDAMANI, I. K.; PATIL, S. A.; RAVIKUMAR, R. L. Relationship of autogamy and self fertility with seed yield per plant and yield components in sunflower (Helianthus annuus L.). Helia, Novi Sad, v.20, , n.26, p.95-102, 1997.

EMBRAPA. Informes da avaliação de genótipos de girassol, 2004/05 e 2005. Londrina, 2006a. 118p. (Embrapa/CNPSo. Documentos, 271)

EMBRAPA. Banco de dados climáticos do Brasil. Campinas: Embrapa Monitoramento por Satélite, 2006. Disponível em: http:/ /www.bdclima.cnpm.embrapa.br>. Acesso em: 06 dez. 2006b.

EMBRAPA. Informes da avaliação de genótipos de girassol, 1996/97 e 1997. Londrina, 1997. 116p. (Embrapa/CNPSo. Documentos, 110)

EMBRAPA. Informes da avaliação de genótipos de girassol, 2002/03 e 2003. Londrina, 2003. 97p. (Embrapa/CNPSo. Documentos, 226).

FALCONER, D. S.; MACKAY, T. F.C. Introduction to quantitative genetics. Harlow: Longman. 1996. 464p.

FARHATULLAH, F.; KHALIL, I. H. Path analysis of the coefficients of sunflower (Helianthus annuus L) hybrids. International Journal of agriculture and Biology, Faisalabad, v.8, n.5, p.621-625, 2006.

FURTADO, M.R.; CRUZ, C.D.; CARDOSO, A.A.; COELHO, A.D.F.; PETERNELLI, L.A. Análise de trilha do rendimento do feijoeiro e seus componentes primários em monocultivo e em consórcio com a cultura do milho. Ciência Rural, Santa Maria, v.32, p.217-220, 2002. 
GOMES, C.N.; CARVALHO, S.P.; JESUS, A.M.S.; CUSTÓDIO, T.N. Caracterização morfoagronômica e coeficientes de trilha de caracteres componentes da produção em mandioca. Pesquisa Agropecuária Brasileira, Brasília, v.42, n.8, p.11211130, 2007.

GOMES, E. M.; UNGARO, M. R. G.; VIEIRA, D. B. Demanda hídrica do girassol (Helianthus annuus L.) obtida em diferentes fases da cultura. In: CONGRESSO LATINOAMERICANO DE HIDRÁULICA, 21., 2004, São Pedro. Anais... São Pedro: IAHR, 2004, 11p

HLADNI, N.;SKORIC, D.; KRALJEVIC-BALALIC, M.; SAKAC, Z.; JOVANOVIC, D. Combining ability for oil content and its correlations with other yield components in sunflower (Helianthus annuus L.). Helia, Novi Sad, v.29, n.44, p.101-110, 2006.

HASSAN, S. W. Heritability, genetic correlations and predicted gains from $S_{1}$ families in two random mated sunflower populations. 2001. 186p. Thesis (Doctor of Philosophy). University of the Punjab, Lahore, Pakistan.

HOERL, A.E.; KENNARD, R.W. Ridge regression: applications to nonorthogonal problems. Technometrics, Washington, v.12, n.1, p.69-82, 1970a.

HOERL, A.E.; KENNARD, R.W. Ridge regression: biased estimation for nonorthogonal problems. Technometrics, Washington, v.12, n.1, p.55-68, $1970 \mathrm{~b}$.

INTERNATIONAL UNION OF PURE AND APPLIED CHEMISTRY. Standard methods for analysis of oils, fats and derivatives. 7.ed. Oxford: Blackwell Scientific Publications,1987.

JOKSIMOVIC, J.; ATLAGIC, J.; SKORIC, D. Path coefficient analysis of some oil yield components in sunflower (Helianthus annuus L.). Helia, Novi Sad, v.22, n.31, p.35-42, 1999.

KHAN, A. Yield performance, heritability and interrelationship in some quantitative traits in sunflower. Helia, Novi Sad, v.24, n.34, p.35-40, 2001.

KNOWLES, P. E. Morphology an anatomy. In: CARTER, J. F. (Ed). Sunflower science and technology. Madison: ASA, 1978. p.55-88.

LAZZAROTTO, J. J.; ROESSING, A. C.; MELlO, H. C. O agronegócio do girassol no mundo e no Brasil. In: LEITE, R. M. V. B.; BRIGHENTI, A. M.; CASTRO, C. (Ed). Girassol no Brasil. Londrina: Embrapa Soja, 2005. p.15-42.

LAKSHAMANRAO, N. G.; SHAMBULINGAPPA, K. G.; KUSUMAKUMARI, P. Studies on path-coefficient analysis in sunflower. In: INTERNATIONAL SUNFLOWER CONFERENCE, 11., 1985, Mar del Plata. Proceedings... Paris: International Sunflower Association, 1985. p.733-735.

MANIVANNAN, N.; MURALIDHARAN, V.; SUBBALAKSHMI, B. Correlation analysis in sunflower. Legume Research, Karnal, v.28, n.1, p.71-73, 2005.
MARQUARDT, D. W. Generalized inverses, ridge regression, biased linear estimation and nonlinear estimation. Technometrics, Washington, v.13, p.591-612, 1970.

NETER, J. Applied linear statistical models. Homewood: Richard D. Irwin, 1974. 842p.

OLIVEIRA, , M. F.; CASTIGLIONI, V. B. R.; CARVALHO, C. G. P. Melhoramento do girassol. In: LEITE, R. M. V. B.; BRIGHENTI, A. M.; CASTRO, C. (Ed). Girassol no Brasil. Londrina: Embrapa Soja, 2005. p.269-297.

PATIL, B.R.; RUDRARADHYA, M.; VIJAYAKUMAR, C. H. M.; BASAPPA, H.; KULKARNI, R. S. Correlation and path analysis in sunflower. Journal Oilseeds Research, v. 13, p.157-161, 1996.

QUAGGIO, J. A.; UNGARO, M. R. G. Girassol. In: VAN RAIJ, B.; CANTARELLA, H.; QUAGGIO, J. A.; FURLANI, A. M. C. (Ed.). Recomendações de adubação e calagem para o Estado de São Paulo. Campinas: IAC, 1997. p.198.

ROMANO, A. B.; VÁZQUEZ, A. N. Origin of the argentine sunflower varieties. Helia, Novi Sad, v.26, n.38, p.127-136, 2003.

SCOTT, A.J., KNOTT, M.A. A cluster analysis method for grouping means in the analysis of variance. Biometrics, Washington, v.30, p.507-512, 1974.

STEEL R. G. D.; TORRIE, J. L. Principles and procedures of statistics. New York: Macgraw-Hill, 1980. 418p.

TEKLEWOLD, A.; JAYARAMAIAH, H.; JAGADEESH, B. N. Correlation and path analysis of physio-morphological characters of sunflower (Helianthus annuus L.) as related to breeding method. Helia, Novi Sad, v.23, n.32, p.105-114, 2000.

TYAGI, A. P. Association and path analysis of yield components and oil percentage in sunflower (Helianthus annuus L.). In: INTERNATIONAL SUNFLOWER CONFERENCE, 11. 1985, Mar del Plata. Proceedings... Paris: International Sunflower Association, 1985. p.807-812.

VIDHYAVATHI, R.; MAHALAKSHMI, P.; MANIVANNAN, N.; MURULIDHARAN, V. Correlation and path analysis in sunflower (Helianthus annuus L.). Agricultural Science Digest, Kernal, v.25, n.1, p.6-10, 2005.

WANI, M. A. Correlation and regression studies in sunflower. Advances in Plant Sciences, Muzaffarnagar, v.17, n.1, p.329332, 2004.

WRIGHT, S. Correlation and causation. Journal Agricultural Research, v.20, p.557-585, 1921. 